\title{
Apparent diffusion coefficient magnetic resonance imaging (ADC-MRI) in the axillary breast cancer lymph node metastasis detection: a narrative review
}

\author{
Camilla De Cataldo ${ }^{1}$, Federico Bruno ${ }^{1}$, Pierpaolo Palumbo ${ }^{1}$, Alessandra Di Sibio ${ }^{2}$, Francesco Arrigoni ${ }^{1}$, \\ Alfredo Clemente ${ }^{3}$, Alberto Bafile ${ }^{4}$, Giovanni Luca Gravina ${ }^{1}$, Salvatore Cappabianca ${ }^{3}$, Antonio Barile ${ }^{1}$, \\ Alessandra Splendiani ${ }^{1}$, Carlo Masciocchi ${ }^{1}$, Ernesto Di Cesare ${ }^{5}$ \\ ${ }^{1}$ Department of Biotechnology and Applied Clinical Sciences, University of L’Aquila, L'Aquila, Italy; ${ }^{2}$ Radiology Unit, San Salvatore Hospital, \\ L’Aquila, Italy; ${ }^{3}$ Department of Precision Medicine, University of Campania “L. Vanvitelli”, Naples, Italy; ${ }^{4}$ Breast Unit, S. Salvatore Hospital, L'Aquila, \\ Italy; ${ }^{5}$ Department of Life, Health and Environmental Sciences, University of L'Aquila, L'Aquila, Italy \\ Contributions: (I) Conception and design: C De Cataldo, F Bruno, C Masciocchi, A Di Sibio; (II) Administrative support: A Barile, A Splendiani, C \\ Masciocchi, E Di Cesare; (III) Provision of study materials or patients: E Di Cesare, P Palumbo, A Clemente, S Cappabianca; (IV) Collection and \\ assembly of data: C De Cataldo, C Masciocchi, A Clemente; (V) Data analysis and interpretation: GL Gravina; (VI) Manuscript writing: All authors; \\ (VII) Final approval of manuscript: All authors. \\ Correspondence to: Federico Bruno. Department of Biotechnology and Applied Clinical Sciences, University of L'Aquila, Via Vetoio 1, 67100, L'Aquila \\ (AQ), Italy. Email: federico.bruno.1988@gmail.com.
}

\begin{abstract}
The presence of axillary lymph nodes metastases in breast cancer is the most significant prognostic factor, with a great impact on morbidity, disease-related survival and management of oncological therapies; for this reason, adequate imaging evaluation is strictly necessary. Physical examination is not enough sensitive to assess breast cancer nodal status; axillary ultrasonography (US) is commonly used to detect suspected or occult nodal metastasis, providing exclusively morphological evaluation, with low sensitivity and positive predictive value. Currently, sentinel lymph node biopsy (SLNB) and/or axillary dissection are the milestone for the diagnostic assessment of axillary lymph node metastases, although its related morbidity. The impact of magnetic resonance imaging (MRI) in the detection of nodal metastases has been widely investigated, as it continues to represent the most promising imaging modality in the breast cancer management. In particular, diffusion-weighted imaging (DWI) and apparent diffusion coefficient (ADC) values represent additional reliable non-contrast sequences, able to improve the diagnostic accuracy of breast cancer MRI evaluation. Several studies largely demonstrated the usefulness of implementing DWI/ADC MRI in the characterization of breast lesions. Herein, in the light of our clinical experience, we perform a review of the literature regarding the diagnostic performance and accuracy of ADC value as potential pre-operative tool to define metastatic involvement of nodal structures in breast cancer patients. For the purpose of this review, PubMed, Web of Science, and SCOPUS electronic databases were searched with different combinations of "axillary lymph node", "breast cancer", "MRI/ADC", "breast MRI" keywords. All original articles, reviews and metanalyses were included.
\end{abstract}

Keywords: Breast cancer; axillary lymph nodes; diffusion-weighted imaging (DWI); apparent diffusion coefficient magnetic resonance imaging (ADC-MRI); metastases

Submitted Jun 03, 2020. Accepted for publication Aug 24, 2020.

doi: $10.21037 /$ gs-20-546

View this article at: http://dx.doi.org/10.21037/gs-20-546 


\section{Introduction}

Breast cancer is the most common cancer among women in the world (1-5). The main causes of cancerrelated death are invasion and metastases. The usual site of spread outside the breast is to the lymph nodes in the axilla $(6,7)$. Axillary lymph node metastasis is one of the most important prognostic factors in breast cancer patients (8). For this reason, the meticulous detection of axillary metastases in newly diagnosed breast cancer is essential for disease staging and treatment decision making (9-14). Unfortunately, the non-invasive differentiation between benign and malignant lymph nodes remains highly problematic, delaying the diagnosis, in case of metastatic risk in lymph nodes, to pathological examination $(15,16)$. The sentinel node biopsy (17) is currently performed during conservative surgery and is eventually followed by axillary dissection. However, up to $70 \%$ of clinically non-metastatic patients are found to be free from malignant axillary disease at dissection and, furthermore, this surgical procedure may determine complications such as lymphedema, arm pain, infection, or seroma $(18,19)$. With magnetic resonance imaging (MRI) $(20,21)$, computed tomography (22), and ultrasonography (23-28), the preoperative study of axillary lymph node status is limited to qualitative and quantitative features such as morphological presentation (cortical thickness, presence of fatty hilum and cortical vascular flow, enhancement patterns, presence of grouping of lymph nodes) (29-31) and measurement of nodal dimensions (maximum long-axis diameter or long/short-axis ratio). All these criteria remain controversial and unsatisfactory for differentiation between metastatic and non-metastatic lymph nodes. We present the following article in accordance with the NARRATIVE REVIEW reporting checklist (available at http://dx.doi. org/10.21037/gs-20-546).

\section{Basic physics of diffusion-weighted imaging (DWI)}

An emerging potential in breast cancer MRI diagnosis is represented by DWI (32). DWI is a functional MRI technique that provides a new contrast mechanism and represents an informative tool about biological microenvironment changes (33). It is characterized by elevated sensitivity in the detection of tissues alterations evaluating the phenomenon known as Brownian motion of free water molecules, which primarily reflects the degree of tissues cellularity (34). Water molecule diffusion is prevented by tissues with a high cellular density, such as tumor tissues, but it is facilitated by a poorly cellular environment containing or by altered cell membranes, especially in case of necrotic lesions. Water motion degree in soft tissue results inversely correlated with tissue cellularity and integrity of cell membranes. Variations of extracellular and intracellular water protons will be reflected by proportional changes in the diffusion coefficient of the tissue, allowing collection of in vivo information about the biophysical properties of tissues. This parameter can be quantitatively defined and derived by DWI images expressing the entity of tissues restriction of diffusion (35). The strong point of DWI compared to dynamic contrast enhanced (DCE)-MRI is its high sensitivity to identify histological changes in the microscopic environment without need for contrast medium administration, which should be avoided in cases of pregnancy and impaired renal function (36-40).

\section{DWI of lymph node metastases: current evidences}

Recent literature reports that DWI with ADC values can be regarded as a useful method in the diagnosis and quantitative measurement of neoplasms (41-43); in particular, several evidences reported significantly lower ADC values in malignancies than in benign lesions and normal tissues $(44,45)$. The ADC value has also been used to predict nodal metastases in many different cancers and to differentiate malignant from benign lymph nodes in various body regions and organs, such as head and neck, uterine, and cervical cancers (46-51). The possible functional mechanism is that high cellularity in lymph node metastases produced by the tumor cells, may decrease extracellular and intracellular spaces, and restrict water molecules mobility, finally leading to a reduction of ADC value (52). The first results about DWI sequences in lymph nodes were shown in patients affected by uterine malignancy. Nakai et al. (53) pointed out that the identification of pelvic pathologic lymph nodes for uterine malignancy is much higher on DWI than conventional T2-weighted (T2W) images. This was related to the clear gap between lymph nodal signal and the surrounding structures such as bowel and vessels. Also, Kim et al. (54) showed that ADC value of pathologic lymph nodes was lower than those of not-pathologic lymph nodes in patients with uterine cervical cancer. On the contrary, Sumi et al. (55) showed that the ADC values 


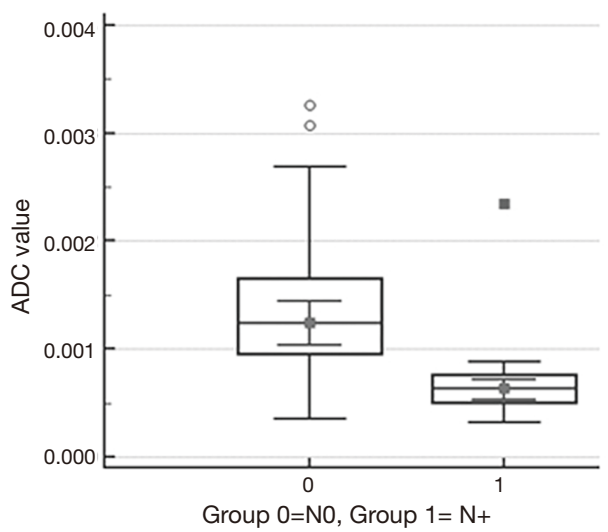

Figure 1 ADC values of metastatic and non-metastatic lymph nodes. ADC values of metastatic lymph nodes were significantly lower than those of non-metastatic lymph nodes $(\mathrm{P}<0.001)$. ADC, apparent diffusion coefficient.

for metastatic lymph nodes in the neck tumors are lower than in non-metastatic, but the resulting differences were not statistically significant. Most of the available studies were focused on cervical lymph nodes in patients with head and neck cancer, and those from pelvic uterine cancer (endometrial and cervical), rectal and prostate; there were reports of similar assessments for oesophageal, lung and abdominal malignancies. Furthermore, other researchers have described no statistically significant differences between ADC values of benign and malignant lymph nodes $(54-63)$. On the contrary, in some studies $(55,64)$ metastatic lymph nodes demonstrate ADC values higher than those not pathologic, phenomena with different results conflicting with those above reported, that may depend on the cytological and histological features of metastases: the presence of necrotic area, inflammation and oedema justifies the lowest tissue cellularity and could increase diffusivity with T2 shine through effect characterized by DWI low signal intensity (65). In addition, in some malignant lymph nodes, benign tissue elements may be present alongside the malignant components, especially in the case of presence of micro-metastases, not possibly highlighted by DWI sequences.

\section{DWI of axillary lymph nodes in breast cancer}

The above-mentioned studies indicated that ADC values of lymph nodes with metastasis were lower compared to those of benign lymph nodes, which may provide a diagnostic value for the evaluation of metastatic lymph node in breast cancer (9). Most part of breast cancer literature focusing on axillary nodal involvement diagnosis shows that, among different studies, lower ADC values were observed in metastatic lymph nodes, and that ADC mean values were statistically significant different between metastatic and non-metastatic axillary lymph nodes $(9,35,46,66-73)$. Yamaguchi et al. (67) in their study showed ADC value of metastatic axillary lymph nodes ranging from $0.553 \times 10^{-3} \mathrm{~mm}^{2} / \mathrm{s}$ to $1.135 \times 10^{-3} \mathrm{~mm}^{2} / \mathrm{s}$ and those of non-metastatic axillary lymph nodes ranged from $0.722 \times 10^{-3} \mathrm{~mm}^{2} / \mathrm{s}$ to $1.739 \times 10^{-3} \mathrm{~mm}^{2} / \mathrm{s}$; Fornasa et al. $(52,66)$ described mean ADC values of metastatic lymph nodes of $0.878 \times 10^{-3} \mathrm{~mm}^{2} / \mathrm{s}$ and mean ADC values of benign lymph nodes of $1.494 \times 10^{-3} \mathrm{~mm}^{2} / \mathrm{s}$. These results are following the majority of authors, who affirm the reliability of DWI in the identification of lymph nodes involved in malignant diseases. This might depend on the relatively high cellularity and rare necrosis in nodal metastases derived from neoplastic mammary epithelial cells. The range of reported values, in other studies, is broad ranging from $0.666 \times 10^{-3} \mathrm{~mm}^{2} / \mathrm{s}$ to $1.369 \times 10^{-3} \mathrm{~mm}^{2} / \mathrm{s}$ $(64,67,69,70)$. In our unpublished experience, we retrospectively evaluated breast MRI performed on a $1.5 \mathrm{~T}$ scanner (Signa Excite, General Electric Medical System, Milwaukee, WI, USA) of 107 patients with newly diagnosed breast cancer who had undergone axillary dissection/ sentinel lymph node biopsy and whose definitive surgical and histological reports were available (median age $51 \pm 6$, 70 patients affected by invasive ductal carcinoma, 28 by invasive lobular carcinoma, 3 by tubular adenocarcinoma and 6 by papillary carcinoma). Two groups were identified according to the nodal status: 50 women with breast carcinoma and metastatic nodal disease and 57 patients with breast carcinoma, but without metastatic axillary lymph nodes involvement. We found that median $\mathrm{ADC}$ value in the metastatic nodal disease group (median $=0.638 \times 10^{-3} \mathrm{~mm}^{2} / \mathrm{s}$ ) was statistically significantly lower than that of the non-metastatic axillary lymph node group $\left(\right.$ median $\left.=1.24 \times 10^{-3} \mathrm{~mm}^{2} / \mathrm{s}\right)(\mathrm{P} \leq 0.001)$ (Figure 1). The area under the receiver operating characteristic (ROC) curve was 0.876 (95\% CI: 0.783-0.939). The sensitivity analysis indicated that an $\mathrm{ADC}$ value of $0.8 \times 10^{-3} \mathrm{~mm}^{2} / \mathrm{s}$ was the optimal cut-off value for the discrimination between metastatic and non-metastatic lymph-nodes (Figures 2,3). This cut-off value obtained a sensitivity, specificity, positive predictive value and negative predictive value of $82.6 \%$, $86 \%, 70.4 \%$ and $92.5 \%$, respectively (Table 1). These results confirm the accuracy and the validity of DWI sequence and 


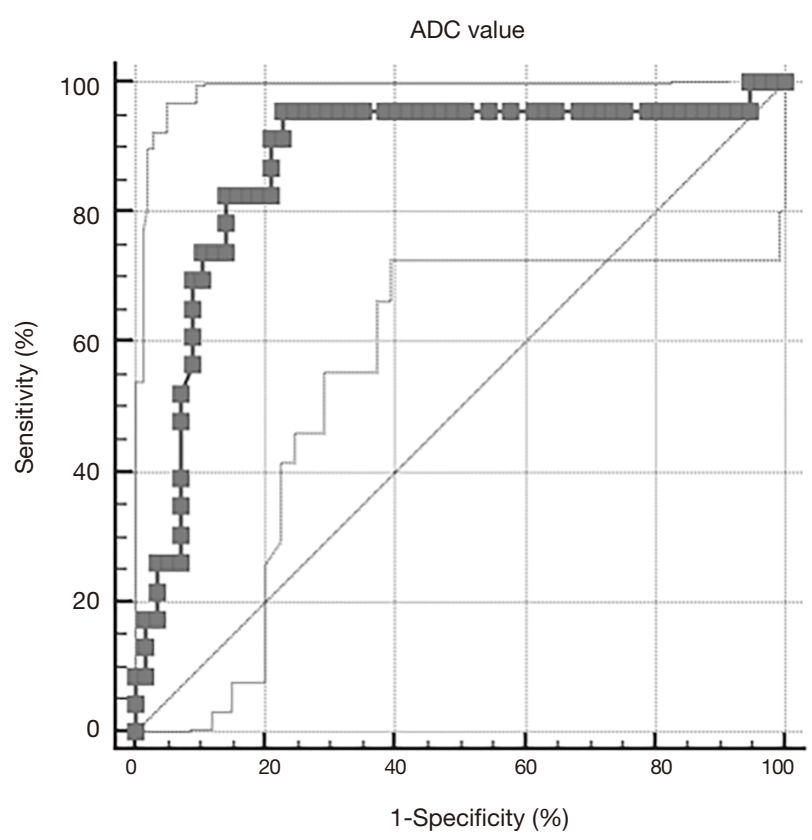

Figure 2 The area under the ROC curve was 0,876 . Using an ADC cut-off value of $0.8 \times 10^{-3} \mathrm{~mm}^{2} / \mathrm{s}$, sensitivity and specificity for diagnosing metastatic axillary lymph node were $82.6 \%$ and $86 \%$ respectively. ROC, receiver operating characteristic; ADC, apparent diffusion coefficient.

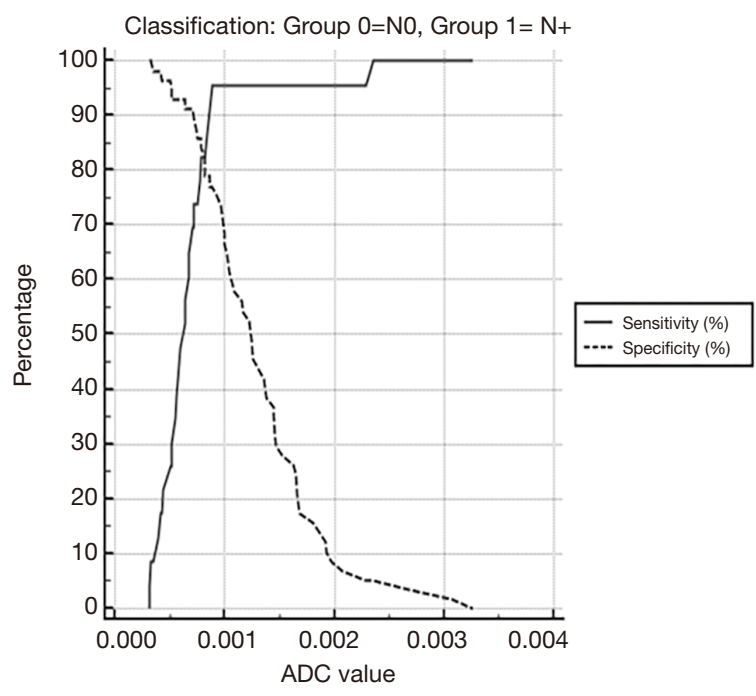

Figure 3 Graphical representation of ADC cut-off value and related specificity and sensitivity. ADC, apparent diffusion coefficient.
Table 1 ADC cut-off value and diagnostic performance

\begin{tabular}{ll}
\hline Parameters & Value \\
\hline Cut-off value (ADC) & $\leq 0.8 \times 10^{-3}$ \\
Sensitivity $(95 \% \mathrm{Cl})$ & $82.61(61.2-95.0)$ \\
Specificity $(95 \% \mathrm{Cl})$ & $85.96(74.2-93.7)$ \\
+PV (95\% Cl) & $70.4(49.8-86.2)$ \\
-PV (95\% Cl) & $92.5(81.8-97.9)$ \\
\hline
\end{tabular}

$+\mathrm{PV}$, positive predictive value; -PV, negative predictive value; $A D C$, apparent diffusion coefficient; $\mathrm{Cl}$, confidence interval.

ADC maps to predict lymph-nodal involvement in breast cancer, and to exclude nodal involvement with high ADC values with high specificity (86\%) and high NPV (92.5\%).

\section{DWI of axillary lymph nodes: technical considerations}

The variability of the data reported in literature may be due to the differences in specimen population, MRI system, different b-values, differences in the use of contrast media and differences in tumor histology. In particular, in a systematic review (43), differences due to country stratified analyses and MRI machine types emerged; ADC values on DWI were apparently decreased in metastatic lymph nodes among China, Italy, and Egypt, while such association was not found in Japan and Korea. One Japanese study shows ADC values of metastatic lymph nodes to be significantly greater than that of the benign ones, probably because of low cellularity of nodal metastases connected with low cellularity primary breast lesions, necrotic areas or oedema (64). Moreover, there is no correlation between decreased ADC values and metastatic lymph nodes when ADC values are calculated on non-Philips 1.5T MRI scanners, while there is no statistically significant difference described in ADC values calculated by Philips 1.5 MRI scanners. These results implied that the MRI scanner technical specifications might affect the association between ADC values and nodal involvement (43). Region of interest (ROI) sampling is another important issue for the assessment of ADC values; in the authors' experience, circular ROIs are placed in the axillary lymph node on DWI, carefully located in the 


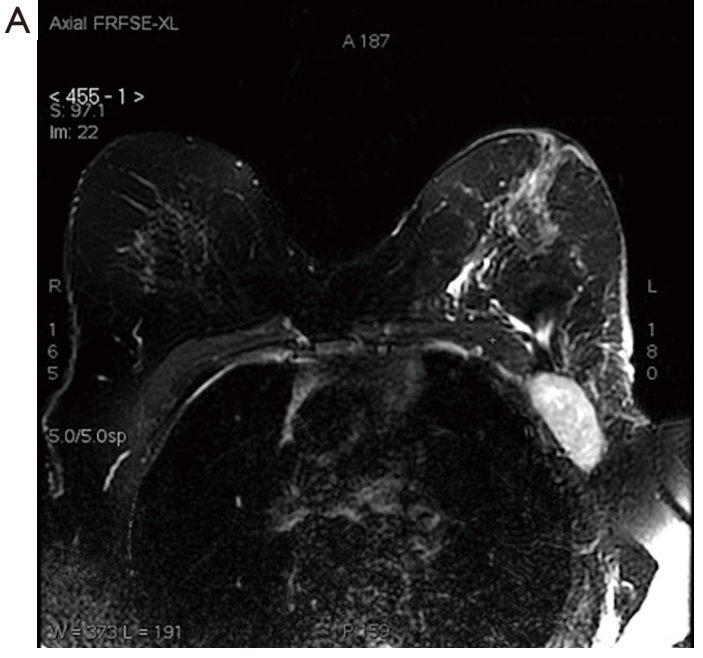

B

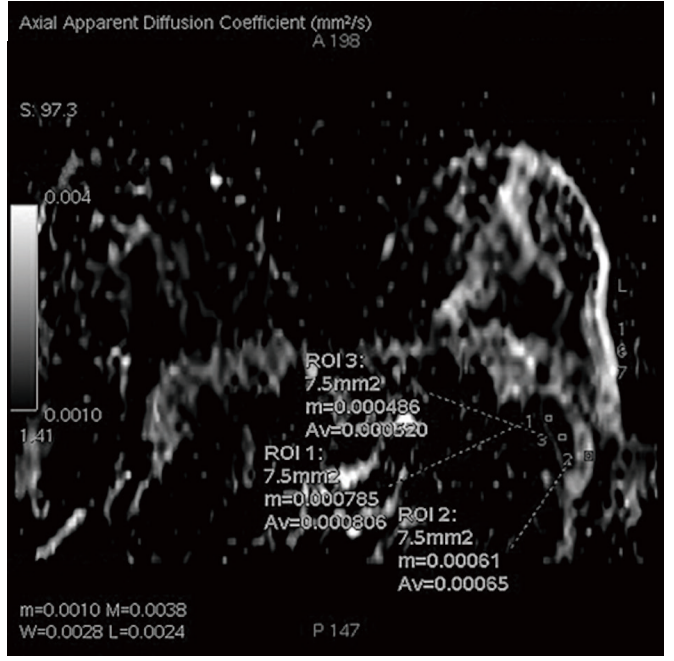

Figure 4 MRI images in patient with breast cancer and metastatic lymph nodes. (A) Axial T2-weighted fat suppression image for the localisation of the lymph node under analysis; (B) ADC map with three ROI positioning; ADC value is obtained by the mean of three measured values. MRI, magnetic resonance imaging; ADC, apparent diffusion coefficient; ROI, region of interest.

central part of the lymph node with normal cortex-medulla ratio or in the central part of the lymph node "package", taking particular care in avoiding necrotic areas, and then copied to the ADC map (Figures 4,5). For an accurate correlation between histological and DWI findings, during the MR images review, the location of the selected lymph node within the axilla is evaluated with the breast surgeon with the support of the operative and histological report

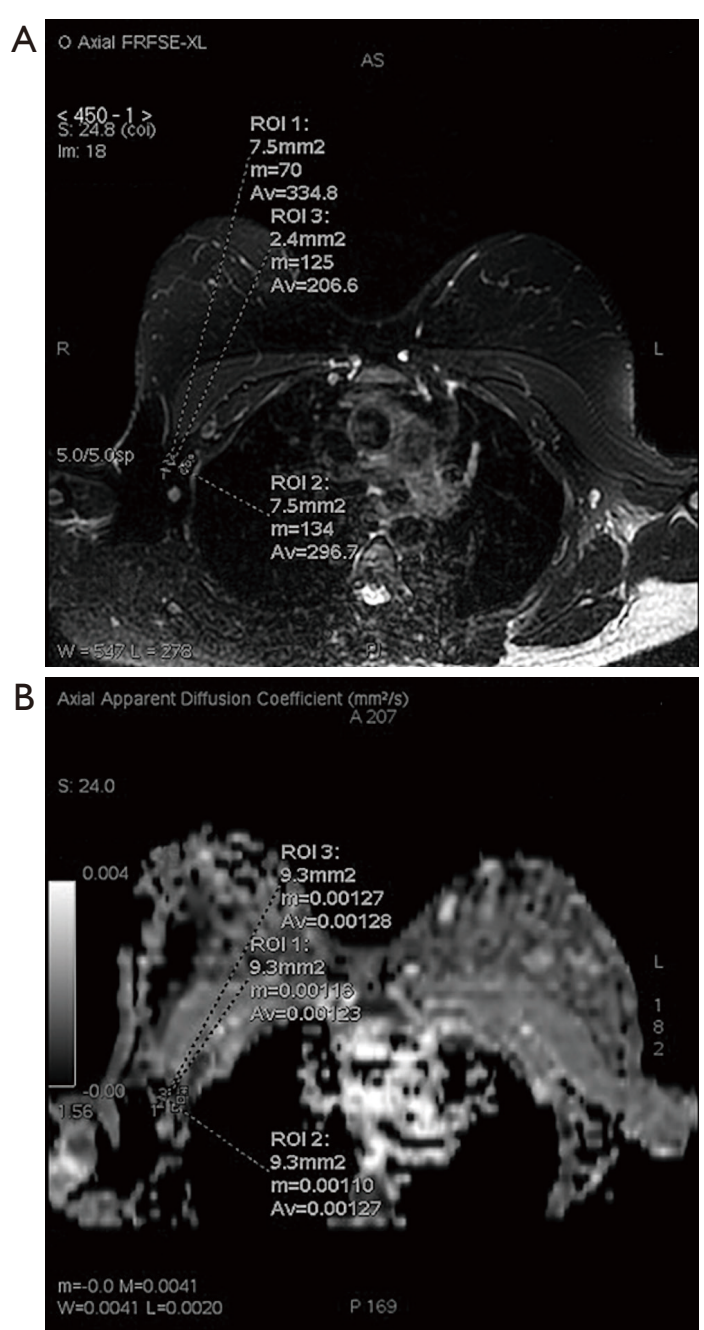

Figure 5 MRI images of a patient with breast carcinoma and benign lymph nodes. (A) Axial T2-weighted fat suppression image for the localisation of the lymph node under analysis; (B) ADC map with three ROI positioning; ADC value is obtained by the mean of three measured values. MRI, magnetic resonance imaging; ADC, apparent diffusion coefficient; ROI, region of interest.

and defined by Berg levels (74). Axillary lymph nodes with short-axis diameter equal to or greater than $4 \mathrm{~mm}$ are included in our analysis. $\mathrm{T} 2 \mathrm{~W}$ fat suppression images are employed to identify involved lymph nodes, as anatomical reference, evaluating size, shape, and depth from the skin surface. Then, the lymph node of interest is identified on DWI through slice by slice comparison of $\mathrm{T} 2 \mathrm{~W}$ fat suppression and DWI. ADC values are calculated using two 
b-values (0 and $\left.800 \mathrm{~s} / \mathrm{mm}^{2}\right)$ through the specific formula: $\mathrm{ADC}=\ln (\mathrm{SI} 1 / \mathrm{SI}) / 800$ in which SI1 and SI2 are signal intensities for $\mathrm{b}=0$ and $800 \mathrm{~s} / \mathrm{mm}^{2}$.

Currently, there is not homogeneity about DWI acquisition timing, whether before (66) or after (67) contrast medium injection. Some studies showed that gadolinium may lead to reduced micro-perfusion and might alter the ADC values (75) while others affirm that gadolinium does not alter tissue diffusion (76-78). In our protocol, DWI images are acquired in the axial plane [free-breathing acquisition, echo planar imaging (EPI) sequence, repetition time (TR)/echo time (TE) 4,000/55.2 ms; acquisition matrix $128 \times 128$; number of excitations (NEX) 8 ; field of view (FOV) $34 \times 34 \mathrm{~cm}$; slice thickness $3 \mathrm{~mm}$ with $0.3 \mathrm{~mm}$ interval, receiver bandwidth $62.5-250$, acceleration factor 2, b values $0 \mathrm{~s} / \mathrm{mm}^{2}$ and $800 \mathrm{~s} / \mathrm{mm}^{2}$ ], before the administration of contrast medium (Gd-BOPTA), to overcome the possible influence of the contrast agent on $\mathrm{ADC}$ values.

Also, different magnetic field intensities and different $b$ values may affect $A D C$ values, creating a not-easily comparable pool of data $(46,64)$.

\section{Conclusions}

DWI sequences are developing rapidly with a progressive technical improvement, especially in spatial resolution and image quality, allowing a more refined morphological assessment (79). The DWI-ADC sequence could be used in the future to avoid axillary dissection in patients with lowintermediate risk of lymph-node involvement (66) as it is strongly related to axillary metastatic involvement; however, technological advances in DWI applicability and protocol standardization are still not ripe to avoid axillary dissection on the basis of ADC results. The goal of the future will be to achieve the least invasive approach to the patient. Some authors showed how DWI could be represent the main element of non-enhanced MRI examination with good sensitivity and high specificity, particularly in the study of mass lesions. According to some authors, DWI sequences could be implemented in non-contrast MRI screening programmes, considering the question of gadoliniumbased contrast agent toxicity and its brain deposits, even in the potential hypothesis of avoiding the double reading (71,80-82). Moreover, the quantitative data derived will in the near future be the most important instrumental finding for radiomics analysis, which with artificial intelligence are revolutionizing the current concept of imaging $(83,84)$.

\section{Acknowledgments}

Funding: None.

\section{Footnote}

Provenance and Peer Review: This article was commissioned by the editorial office, Gland Surgery for the series "Multimodality Advanced Imaging and Intervention in Gland Diseases". The article has undergone external peer review.

Reporting Checklist: The authors have completed the NARRATIVE REVIEW reporting checklist. Available at http://dx.doi.org/10.21037/gs-20-546

Conflicts of Interest: All authors have completed the ICMJE uniform disclosure form (available at http://dx.doi. org/10.21037/gs-20-546). The series "Multimodality Advanced Imaging and Intervention in Gland Diseases" was commissioned by the editorial office without any funding or sponsorship. Antonio Barile served as the unpaid Guest Editor of the series. The other authors have no other conflicts of interest to declare.

Ethical Statement: The authors are accountable for all aspects of the work in ensuring that questions related to the accuracy or integrity of any part of the work are appropriately investigated and resolved.

Open Access Statement: This is an Open Access article distributed in accordance with the Creative Commons Attribution-NonCommercial-NoDerivs 4.0 International License (CC BY-NC-ND 4.0), which permits the noncommercial replication and distribution of the article with the strict proviso that no changes or edits are made and the original work is properly cited (including links to both the formal publication through the relevant DOI and the license). See: https://creativecommons.org/licenses/by-nc-nd/4.0/.

\section{References}

1. Siegel R, Naishadham D, Jemal A. Cancer statistics, 2012. CA Cancer J Clin 2012;62:10-29. 
2. Trimboli RM, Codari M, Bert A, et al. Breast arterial calcifications on mammography: intra- and inter-observer reproducibility of a semi-automatic quantification tool. Radiol Med 2018;123:168-73.

3. Woelfel IA, Fernandez LJ, Idowu MO, et al. A high burden of comorbid conditions leads to decreased survival in breast cancer. Gland Surg 2018;7:216-27.

4. Chatterjee A. Long term effects of modern breast cancer surgery. Gland Surg 2018;7:366-70.

5. Renzulli M, Zanotti S, Clemente A, et al. Hereditary breast cancer: screening and risk reducing surgery. Gland Surg 2019;8:S142-9.

6. Trignani M, Argenone A, Di Biase S, et al. Interobserver variability of clinical target volume delineation in definitive radiotherapy of neck lymph node metastases from unknown primary. A cooperative study of the Italian Association of Radiotherapy and Clinical Oncology (AIRO) Head and Neck Group. Radiol Med 2019;124:682-92.

7. Murphy BL, Fazzio RT, Hoskin TL, et al. Management of the axilla in metaplastic breast carcinoma. Gland Surg 2018;7:200-6.

8. Lewis RS, George A, Rusby JE. Nipple-sparing mastectomy in women at high risk of developing breast cancer. Gland Surg 2018;7:325-36.

9. Luo N, Su D, Jin G, et al. Apparent diffusion coefficient ratio between axillary lymph node with primary tumor to detect nodal metastasis in breast cancer patients. J Magn Reson Imaging 2013;38:824-8.

10. Bednarova I, Londero V, Linda A, et al. Do clinical and radiologic features help predict malignancy of $\mathrm{B} 3$ breast lesions without epithelial atypia (B3a)? Radiol Med 2018;123:809-17.

11. Fiorentino A, Gregucci F, Mazzola R, et al. Intensitymodulated radiotherapy and hypofractionated volumetric modulated arc therapy for elderly patients with breast cancer: comparison of acute and late toxicities. Radiol Med 2019;124:309-14.

12. Amato F, Bicchierai G, Cirone D, et al. Preoperative loco-regional staging of invasive lobular carcinoma with contrast-enhanced digital mammography (CEDM). Radiol Med 2019;124:1229-37.

13. Ramasubramanian V, Balaji K, Balaji Subramanian S, et al. Hybrid volumetric modulated arc therapy for whole breast irradiation: a dosimetric comparison of different arc designs. Radiol Med 2019;124:546-54.

14. Mitchell SD, Willey SC, Beitsch P, et al. Evidence based outcomes of the American Society of Breast Surgeons Nipple Sparing Mastectomy Registry. Gland Surg
2018;7:247-57.

15. Mansel RE, Fallowfield L, Kissin M, et al. Randomized multicenter trial of sentinel node biopsy versus standard axillary treatment in operable breast cancer: the ALMANAC Trial. J Natl Cancer Inst 2006;98:599-609.

16. Petralia G, Padhani AR, Pricolo P, et al. Wholebody magnetic resonance imaging (WB-MRI) in oncology: recommendations and key uses. Radiol Med 2019;124:218-33.

17. Özel D, Aydin T. A clinical compilation of lymph node pathologies comparing the diagnostic performance of biopsy methods. J Ultrasound 2019;22:59-64.

18. Blanchard DK, Donohue JH, Reynolds C, et al. Relapse and morbidity in patients undergoing sentinel lymph node biopsy alone or with axillary dissection for breast cancer. Arch Surg 2003;138:482-7; discussion 487-8.

19. Gillespie TC, Sayegh HE, Brunelle CL, et al. Breast cancer-related lymphedema: risk factors, precautionary measures, and treatments. Gland Surg 2018;7:379-403.

20. Trimboli RM, Codari M, Khouri Chalouhi K, et al. Correlation between voxel-wise enhancement parameters on DCE-MRI and pathological prognostic factors in invasive breast cancers. Radiol Med 2018;123:91-7.

21. Macchini M, Ponziani M, Iamurri AP, et al. Role of DCEMR in predicting breast cancer subtypes. Radiol Med 2018;123:753-64.

22. Travieso-Aja MDM, Maldonado-Saluzzi D, NaranjoSantana P, et al. Diagnostic performance of contrastenhanced dual-energy spectral mammography (CESM): a retrospective study involving 644 breast lesions. Radiol Med 2019;124:1006-17.

23. Zanotel M, Bednarova I, Londero V, et al. Automated breast ultrasound: basic principles and emerging clinical applications. Radiol Med 2018;123:1-12.

24. Bartolotta TV, Orlando A, Cantisani V, et al. Focal breast lesion characterization according to the BI-RADS US lexicon: role of a computer-aided decision-making support. Radiol Med 2018;123:498-506.

25. Zhang X, Chen J, Zhou Y, et al. Diagnostic value of an automated breast volume scanner compared with a hand-held ultrasound: a meta-analysis. Gland Surg 2019;8:698-711.

26. Di Segni M, de Soccio V, Cantisani V, et al. Automated classification of focal breast lesions according to S-detect: validation and role as a clinical and teaching tool. J Ultrasound 2018;21:105-18.

27. Carlino G, Rinaldi P, Giuliani M, et al. Ultrasound-guided preoperative localization of breast lesions: a good choice. J 
Ultrasound 2019;22:85-94.

28. Alikhassi A, Azizi F, Ensani F. Imaging features of granulomatous mastitis in 36 patients with new sonographic signs. J Ultrasound 2020;23:61-8.

29. Ahn HS, Jang M, Kim SM, et al. Usefulness of preoperative breast magnetic resonance imaging with a dedicated axillary sequence for the detection of axillary lymph node metastasis in patients with early ductal breast cancer. Radiol Med 2019;124:1220-8.

30. Liu G, Zhang MK, He Y, et al. Shear wave elasticity of breast lesions: would it be correlated with the extracellular matrix components? Gland Surg 2019;8:399-406.

31. Liu G, Zhang MK, He Y, et al. BI-RADS 4 breast lesions: could multi-mode ultrasound be helpful for their diagnosis? Gland Surg 2019;8:258-70.

32. Yoshida T, Urikura A, Shirata K, et al. Short tau inversion recovery in breast diffusion-weighted imaging: signalto-noise ratio and apparent diffusion coefficients using a breast phantom in comparison with spectral attenuated inversion recovery. Radiol Med 2018;123:296-304.

33. Beyhan M, Sade R, Koc E, et al. The evaluation of prostate lesions with IVIM DWI and MR perfusion parameters at 3T MRI. Radiol Med 2019;124:87-93.

34. Fogante M, Tagliati C, De Lisa M, et al. Correlation between apparent diffusion coefficient of magnetic resonance imaging and tumor-infiltrating lymphocytes in breast cancer. Radiol Med 2019;124:581-7.

35. Yilmaz E, Erok B, Atca AO. Measurement of apparent diffusion coefficient in discrimination of benign and malignant axillary lymph nodes. Pol J Radiol 2019;84:e592-7.

36. Cipolla V, Santucci D, Guerrieri D, et al. Correlation between 3T apparent diffusion coefficient values and grading of invasive breast carcinoma. Eur J Radiol 2014;83:2144-50.

37. Giannitto C, Campoleoni M, Maccagnoni S, et al. Unindicated multiphase CT scans in non-traumatic abdominal emergencies for women of reproductive age: a significant source of unnecessary exposure. Radiol Med 2018;123:185-90.

38. Paolicchi F, Bastiani L, Guido D, et al. Radiation dose exposure in patients affected by lymphoma undergoing repeat CT examinations: how to manage the radiation dose variability. Radiol Med 2018;123:191-201.

39. Patel A, Jackson B. Low-dose radiation use in diagnostic imaging and cancer therapy settings. Radiol Med 2018;123:618-9.

40. Tomà $\mathrm{P}$, Bartoloni $\mathrm{A}$, Salerno $\mathrm{S}$, et al. Protecting sensitive patient groups from imaging using ionizing radiation: effects during pregnancy, in fetal life and childhood. Radiol Med 2019;124:736-44.

41. Park SH, Moon WK, Cho N, et al. Diffusion-weighted MR imaging: pretreatment prediction of response to neoadjuvant chemotherapy in patients with breast cancer. Radiology 2010;257:56-63.

42. Downey K, Riches SF, Morgan VA, et al. Relationship between imaging biomarkers of stage I cervical cancer and poor-prognosis histologic features: quantitative histogram analysis of diffusion-weighted MR images. AJR Am J Roentgenol 2013;200:314-20.

43. Sun JH, Jiang L, Guo F, et al. Diagnostic significance of apparent diffusion coefficient values with diffusion weighted MRI in breast cancer: a meta- analysis. Asian Pac J Cancer Prev 2014;15:8271-7.

44. Jeh SK, Kim SH, Kim HS, et al. Correlation of the apparent diffusion coefficient value and dynamic magnetic resonance imaging findings with prognostic factors in invasive ductal carcinoma. J Magn Reson Imaging 2011;33:102-9.

45. Woodhams R, Ramadan S, Stanwell P, et al. Diffusionweighted imaging of the breast: principles and clinical applications. Radiographics 2011;31:1059-84.

46. Kim EJ, Kim SH, Kang BJ, et al. Diagnostic value of breast MRI for predicting metastatic axillary lymph nodes in breast cancer patients: diffusion-weighted MRI and conventional MRI. Magn Reson Imaging 2014;32:1230-6.

47. Vogl TJ, Albrecht MH, Nour-Eldin NA, et al. Assessment of salivary gland tumors using MRI and CT: impact of experience on diagnostic accuracy. Radiol Med 2018;123:105-16.

48. Beigi M, Kazerooni AF, Safari M, et al. Heterogeneity analysis of diffusion-weighted MRI for prediction and assessment of microstructural changes early after one cycle of induction chemotherapy in nasopharyngeal cancer patients. Radiol Med 2018;123:36-43.

49. Vidiri A, Minosse S, Piludu F, et al. Cervical lymphadenopathy: can the histogram analysis of apparent diffusion coefficient help to differentiate between lymphoma and squamous cell carcinoma in patients with unknown clinical primary tumor? Radiol Med 2019;124:19-26.

50. Woo S, Kim SY, Cho JY, et al. Apparent diffusion coefficient for prediction of parametrial invasion in cervical cancer: a critical evaluation based on stratification to a Likert scale using T2-weighted imaging. Radiol Med 2018;123:209-16. 
51. Yildiz S, Aralasmak A, Yetis H, et al. MRI findings and utility of DWI in the evaluation of solid parathyroid lesions. Radiol Med 2019;124:360-7.

52. Fornasa F, Pinali L, Gasparini A, et al. Diffusion-weighted magnetic resonance imaging in focal breast lesions: analysis of 78 cases with pathological correlation. Radiol Med 2011;116:264-75.

53. Nakai G, Matsuki M, Inada Y, et al. Detection and evaluation of pelvic lymph nodes in patients with gynecologic malignancies using body diffusion-weighted magnetic resonance imaging. J Comput Assist Tomogr 2008;32:764-8.

54. Kim JK, Kim KA, Park BW, et al. Feasibility of diffusionweighted imaging in the differentiation of metastatic from nonmetastatic lymph nodes: early experience. J Magn Reson Imaging 2008;28:714-9.

55. Sumi M, Sakihama N, Sumi T, et al. Discrimination of metastatic cervical lymph nodes with diffusion-weighted MR imaging in patients with head and neck cancer. AJNR Am J Neuroradiol 2003;24:1627-34.

56. Akduman EI, Momtahen AJ, Balci NC, et al. Comparison between malignant and benign abdominal lymph nodes on diffusion-weighted imaging. Acad Radiol 2008;15:641-6.

57. Chen YB, Liao J, Xie R, et al. Discrimination of metastatic from hyperplastic pelvic lymph nodes in patients with cervical cancer by diffusion-weighted magnetic resonance imaging. Abdom Imaging 2011;36:102-9.

58. de Bondt RB, Hoeberigs MC, Nelemans PJ, et al. Diagnostic accuracy and additional value of diffusionweighted imaging for discrimination of malignant cervical lymph nodes in head and neck squamous cell carcinoma. Neuroradiology 2009;51:183-92.

59. Sakurada A, Takahara T, Kwee TC, et al. Diagnostic performance of diffusion-weighted magnetic resonance imaging in esophageal cancer. Eur Radiol 2009;19:1461-9.

60. Sardanelli F, Podo F, Santoro F, et al. Multicenter surveillance of women at high genetic breast cancer risk using mammography, ultrasonography, and contrastenhanced magnetic resonance imaging (the high breast cancer risk italian 1 study): final results. Invest Radiol 2011;46:94-105.

61. Sardanelli F, Podo F, D'Agnolo G, et al. Multicenter comparative multimodality surveillance of women at genetic-familial high risk for breast cancer (HIBCRIT study): interim results. Radiology 2007;242:698-715.

62. Park SO, Kim JK, Kim KA, et al. Relative apparent diffusion coefficient: determination of reference site and validation of benefit for detecting metastatic lymph nodes in uterine cervical cancer. J Magn Reson Imaging 2009;29:383-90.

63. Pauls S, Schmidt SA, Juchems MS, et al. Diffusionweighted MR imaging in comparison to integrated [(1) (8)F]-FDG PET/CT for $\mathrm{N}$-staging in patients with lung cancer. Eur J Radiol 2012;81:178-82.

64. Kamitani T, Hatakenaka M, Yabuuchi H, et al. Detection of axillary node metastasis using diffusion-weighted MRI in breast cancer. Clin Imaging 2013;37:56-61.

65. Perri M, Grattacaso G, di Tunno V, et al. T2 shinethrough phenomena in diffusion-weighted MR imaging of lumbar discs after oxygen-ozone discolysis: a randomized, double-blind trial with steroid and $\mathrm{O} 2-\mathrm{O} 3$ discolysis versus steroid only. Radiol Med 2015;120:941-50.

66. Fornasa F, Nesoti MV, Bovo C, et al. Diffusion-weighted magnetic resonance imaging in the characterization of axillary lymph nodes in patients with breast cancer. J Magn Reson Imaging 2012;36:858-64.

67. Yamaguchi K, Schacht D, Nakazono T, et al. Diffusion weighted images of metastatic as compared with nonmetastatic axillary lymph nodes in patients with newly diagnosed breast cancer. J Magn Reson Imaging 2015;42:771-8.

68. Scaranelo AM, Eiada R, Jacks LM, et al. Accuracy of unenhanced MR imaging in the detection of axillary lymph node metastasis: study of reproducibility and reliability. Radiology 2012;262:425-34.

69. Nakai G, Matsuki M, Harada T, et al. Evaluation of axillary lymph nodes by diffusion-weighted MRI using ultrasmall superparamagnetic iron oxide in patients with breast cancer: initial clinical experience. J Magn Reson Imaging 2011;34:557-62.

70. He N, Xie C, Wei W, et al. A new, preoperative, MRIbased scoring system for diagnosing malignant axillary lymph nodes in women evaluated for breast cancer. Eur J Radiol 2012;81:2602-12.

71. Iima M, Honda M, Sigmund EE, et al. Diffusion MRI of the breast: Current status and future directions. J Magn Reson Imaging 2020;52:70-90.

72. Schipper RJ, Paiman ML, Beets-Tan RG, et al. Diagnostic Performance of Dedicated Axillary T2- and Diffusionweighted MR Imaging for Nodal Staging in Breast Cancer. Radiology 2015;275:345-55.

73. Chung J, Youk JH, Kim JA, et al. Role of diffusionweighted MRI: predicting axillary lymph node metastases in breast cancer. Acta Radiol 2014;55:909-16.

74. Attene F, Paliogiannis P, Scognamillo F, et al. Axillary lymph node dissection in patients with breast cancer and 
sentinel lymph node micrometastasis or isolated tumor cells: is it necessary? Tumori 2012;98:320-3.

75. Yuen S, Yamada K, Goto M, et al. Microperfusion-induced elevation of ADC is suppressed after contrast in breast carcinoma. J Magn Reson Imaging 2009;29:1080-4.

76. Chen G, Jespersen SN, Pedersen M, et al. Intravenous administration of Gd-DTPA prior to DWI does not affect the apparent diffusion constant. Magn Reson Imaging 2005;23:685-9.

77. Fitzek C, Mentzel HJ, Fitzek S, et al. Echoplanar diffusion-weighted MRI with intravenous gadoliniumDTPA. Neuroradiology 2003;45:592-7.

78. Ogura A, Hayakawa K, Miyati T, et al. The effect of susceptibility of gadolinium contrast media on diffusionweighted imaging and the apparent diffusion coefficient. Acad Radiol 2008;15:867-72.

79. Sardanelli F, Carbonaro LA, Montemezzi S, et al. Clinical Breast MR Using MRS or DWI: Who Is the Winner? Front Oncol 2016;6:217.

80. Splendiani A, Perri M, Marsecano C, et al. Effects of

Cite this article as: De Cataldo C, Bruno F, Palumbo P, Di Sibio A, Arrigoni F, Clemente A, Bafile A, Gravina GL, Cappabianca S, Barile A, Splendiani A, Masciocchi C, Di Cesare E. Apparent diffusion coefficient magnetic resonance imaging (ADC-MRI) in the axillary breast cancer lymph node metastasis detection: a narrative review. Gland Surg 2020;9(6):2225-2234. doi: $10.21037 / g s-20-546$ serial macrocyclic-based contrast materials gadoterate meglumine and gadobutrol administrations on gadoliniumrelated dentate nuclei signal increases in unenhanced T1weighted brain: a retrospective study in 158 multiple sclerosis (MS) patients. Radiol Med 2018;123:125-34.

81. Splendiani A, Corridore A, Torlone S, et al. Visible T1hyperintensity of the dentate nucleus after multiple administrations of macrocyclic gadolinium-based contrast agents: yes or no? Insights Imaging 2019;10:82.

82. Pasquini L, Rossi Espagnet MC, Napolitano A, et al. Dentate nucleus T1 hyperintensity: is it always gadolinium all that glitters? Radiol Med 2018;123:469-73.

83. Grassi R, Miele V, Giovagnoni A. Artificial intelligence: a challenge for third millennium radiologist. Radiol Med 2019;124:241-2.

84. Filograna L, Lenkowicz J, Cellini F, et al. Identification of the most significant magnetic resonance imaging (MRI) radiomic features in oncological patients with vertebral bone marrow metastatic disease: a feasibility study. Radiol Med 2019;124:50-7. 